\title{
Exposure-related, global alterations in innate and adaptive immunity; a consideration for re-use of non-human primates in research
}

\author{
François A Bates ${ }^{1}$, Elizabeth H Duncan ${ }^{2}$, Monika Simmons ${ }^{3}$, Tanisha Robinson ${ }^{2,4}$, Sridhar Samineni ${ }^{1,5}$, Natasa \\ Strbo $^{6}$, Eileen Villasante ${ }^{7}$, Elke Bergmann-Leitner ${ }^{2}$, Wathsala Wijayalath ${ }^{\text {Corresp. } 7,8}$

Background Non-human primates (NHPs) play an important role in biomedical research, where they are often being re-used in multiple research studies over the course of their life-time. Researchers employ various study-specific screening criteria to reduce potential variables associated with subsequent re-use of NHPs. However, criteria set for NHP re-assignments largely neglect the impact of previous exposures on overall biology. Since the immune system is a key determinant of overall biological outcome, an altered biological state could be predicted by monitoring global changes in the immune profile. We postulate that every different exposure or a condition can generate a unique global immune profile in NHPS.

Methods Changes in the global immune profile were evaluated in three different groups of rhesus macaques previously enrolled in dengue or malaria vaccine studies over six months after their last exposure. Naïve animals served as the baseline. Fresh blood samples were stained with various immune cell surface markers and analyzed by multi-color flow-cytometry to study immune cell dynamics in the peripheral blood. Serum cytokine profile in the pre-exposed animals were analyzed by mesoscale assay using a customized U-PLEX NHP biomarker panel of 12 cytokines/chemokines.

Results Pre-exposed macaques showed altered dynamics in circulating cytokines and certain innate and adaptive immune cell subsets such as monocytes, HLA-DR+NKT cells, B cells and T cells. Some of these changes were transient, while some lasted for more than six months. Each group seemed to develop a global immune profile unique to their particular exposure.

Conclusion Our data strongly suggest that re-used NHPs should be evaluated for long-term, overall immunological changes and randomly assigned to new studies to avoid study bias. 


\section{Exposure-related, global alterations in innate and} 2 adaptive immunity; a consideration for re-use of non3 human primates in research.

4

5

6

7

8

9

10

11

12

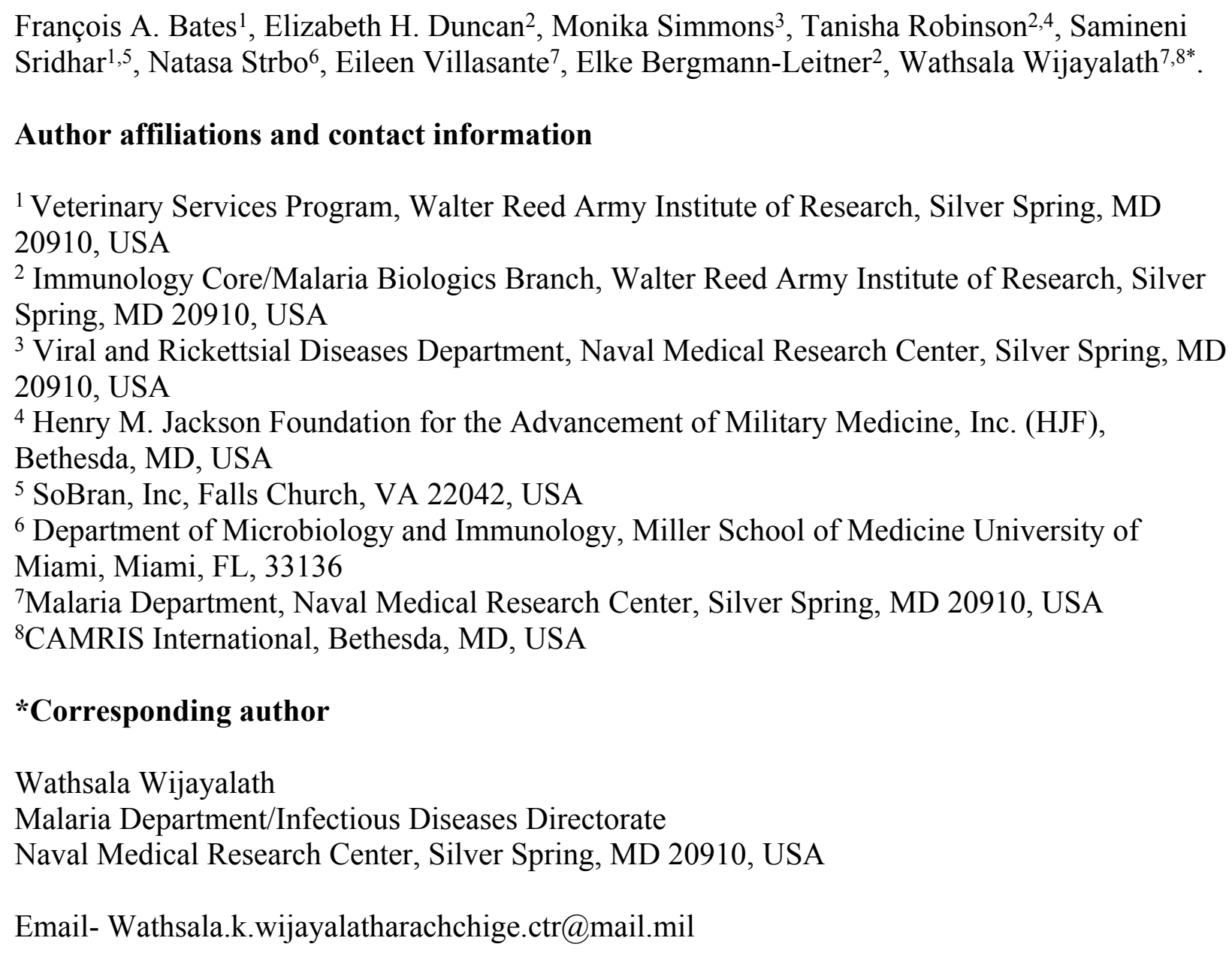


46

47

48

49

50

51

52

53

54

55

56

57

58

59

60

61

62

63

64

65

66

67

68

69

75

76

77

78

79

80

81

82

83

84

\section{Abstract}

\section{Background}

Non-human primates (NHPs) play an important role in biomedical research, where they are often being re-used in multiple research studies over the course of their life-time. Researchers employ various study-specific screening criteria to reduce potential variables associated with subsequent re-use of NHPs. However, criteria set for NHP re-assignments largely neglect the impact of previous exposures on overall biology. Since the immune system is a key determinant of overall biological outcome, an altered biological state could be predicted by monitoring global changes in the immune profile. We postulate that every different exposure or a condition can generate a unique global immune profile in NHPs.

\section{Methods}

Changes in the global immune profile were evaluated in three different groups of rhesus macaques previously enrolled in dengue or malaria vaccine studies over six months after their last exposure. Naïve animals served as the baseline. Fresh blood samples were stained with various immune cell surface markers and analyzed by multi-color flow-cytometry to study immune cell dynamics in the peripheral blood. Serum cytokine profile in the pre-exposed animals were analyzed by mesoscale assay using a customized U-PLEX NHP biomarker panel of 12 cytokines/chemokines.

\section{Results}

Pre-exposed macaques showed altered dynamics in circulating cytokines and certain innate and adaptive immune cell subsets such as monocytes, HLA-DR+NKT cells, B cells and T cells. Some of these changes were transient, while some lasted for more than six months. Each group seemed to develop a global immune profile unique to their particular exposure.

\section{Conclusion}

Our data strongly suggest that re-used NHPs should be evaluated for long-term, overall immunological changes and randomly assigned to new studies to avoid study bias.

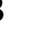

(5)


85

86

87

88

89

90

91

92

93

94

95

96

97

98

99

100

101

102

103

104

105

106

107

108

109

110

111

112

113

114

115

116

117

118

119

120

121

122

123

124

125

126

127

128

129

130

\section{Introduction}

Non-human primates (NHPs) are a long-lived, sentient species which can be trained to perform certain cognitive and behavioral tasks (Scott et al., 2003; Prescott, Bowell \& Buchanan-Smith, 2004; Calapai et al., 2017). Most of the experiments involving NHPs are not terminal, therefore, they remain available in research facilities after the study end-points. For these reasons, it is a common practice to re-use NHPs in multiple research studies over the course of their lifetime (Tardif, et al., 2013; Epstein \& Vermeire, 2017). A survey conducted in North America from 2010 to 2012, showed that of the 26 facilities that responded to the survey, 5 facilities re-used $80 \%$ or more NHPs and 7 facilities re-used $50 \%$ or more NHPs within their own facility (Lankau et al., 2014). An earlier analysis of 2,937 peer-reviewed articles published in 2001 had determined that $14.4 \%$ of the studies re-used or would re-use NHPs in various experiments (Carlsson et al., 2004). Their limited availability, ethical constraints, high cost of acquisition and maintenance can further influence repeated use of NHPs in research.

When re-assigning NHPs in various research protocols, each study typically implements their own study-specific, screening criteria to exclude previously exposed animals to a similar disease or similar environmental stimulus. As a standard practice, in most cases, NHPs are re-assigned to new studies after a short resting period. There have been no reports clearly defining the scientific basis of length of the resting period between repeated exposures of NHPs to different study protocols. Majority of NHP re-assignments assume that effect of pre-exposure can only be specific to a particular study or a disease, mostly neglecting their impact on overall biology of the animals. Since the immune system is a key determinant of overall biological outcome, changes in the global immune profile could represent an altered biological state of the animal. Here, we propose that global changes in immune system thus, could be a useful inclusion/exclusion criteria to re-use NHPs in different protocols or to determine their resting period.

The immune system continuously cross-talks with the rest of the organs in the body, modulating the immune profile accordingly, not only during diseases directly affecting the immune system, but in diseases related to other systems such as neurological or reproductive system (Bell et al., 2011; Blair et al., 2016; Raper et al., 2016). Likewise, the critical role of non-specific immunomodulatory effects induced by vaccines targeting infectious diseases have been increasingly recognized over the recent years (Kandasamy et al., 2016; de Bree et al., 2018; Blok et al., 2020). It has been shown that exposure to vaccines such as Bacille Calmette-Guerin (BCG) and small pox can induce long-lasting, heterologous immune effects mediated by epigenetic modifications in innate immune cells (trained immunity) (Benn et al., 2013; Blok et al., 2015; Arts et al., 2016; Neeta et al., 2020). Acute viral or bacterial infections also seem to induce global changes in immune responses over a considerable period of time (Silveira et al., 2017; Raijmakers et al., 2019). Rhesus macaques experimentally infected with Zika virus have been shown to maintain altered frequencies of circulating monocyte and myeloid dendritic cell subsets up to seventy days post-infection (Silveira et al., 2017). During a six month follow-up, patients with acute $\mathrm{Q}$ fever have demonstrated long-lasting transcriptional changes in circulating monocytes and certain cytokines (Raijmakers et al., 2019). In fact, various factors such as transportation, relocation, acclimation, social conditions and aging also can adversely impact the immune profile of macaques (Didier et al., 2012; Capitanio \& Cole, 2015; Nehete et al., 2017). 
131 These evidence lead to our hypothesis that every different exposure or a condition can generate a

132

133

134

135

136

137

138

139

140

141

142

143

144

145

146

147

148

149

150

151

152

153

154

155

156

157

158

159

160

161

162

163

164

165

166

167

168

169

170

171

172

173

174

175

176 unique global immune profile in NHPs.

In order to understand whether each exposure could have resulted in a distinctive overall immune profile, we studied three different groups of rhesus macaques previously enrolled in infectious disease research protocols. Pre-exposed animals were monitored over 6 months after their most recent exposure, whereas naïve macaques served as the baseline. To avoid any technical bias, flow cytometric monitoring of the frequency of leukocyte populations and automated leukocyte counting was made in whole blood. We found long-term, exposure-related changes in circulating immune cell subsets and cytokines, leading to distinctive overall immune profiles in pre-exposed rhesus macaques.

\section{Materials and Methods}

\section{Animals}

Blood and serum samples reported in the present study were received as part of a tissue-sharing program from the protocols reviewed and approved by Walter Reed Army Institute of Research (WRAIR)/Naval Medical Research Center (NMRC) Institutional Animal Care and Use Committee in compliance with all applicable federal regulations governing the protection of animals and research (WRAIR/NMRC IACUC protocols:18-IDD-01L, 18-VD-27L, 18-VET25L. For samples shared through WRAIR clinical pathology laboratory, 18-VET-24L). Experiments reported herein was carried out in compliance with the Animal Welfare Act and per the principles set forth in the "Guide for Care and Use of Laboratory Animals," Institute of Laboratory Animals Resources, National Research Council, National Academy Press, 2011, the Public Health Service Animal Welfare Policy, and the policies of WRAIR. Adult purpose-bred rhesus macaques of Indian origin were housed at the WRAIR animal facility for the duration of their studies. Animals were socially pair-housed and fed a commercial diet (Lab diet 5038, Purina Mills International), provided free access to water, and supplemented with a variety of fresh fruits and vegetables. Environmental enrichment was provided in accordance with standard operating procedures of the WRAIR animal facility. Animal cages were cleaned daily and sanitized bimonthly. Automatic lighting was provided through a 12:12 hour cycle.

\section{Experimental and control groups}

Three groups of rhesus macaques (Macaca mulatta, three to six years old) were selected based on their most recent study exposures. Since all the assessments were made on fresh whole blood samples, age matched, naïve rhesus macaques $(n=9$, Females $(F)=3$, Males $(M)=6)$ served as the control group for all the comparisons described in the present study. Experimental group one consisted of rhesus macaques ( $\mathrm{n}=10, \mathrm{~F}=2, \mathrm{M}=8$ ) that had been primed with bivalent or tetravalent dengue purified inactivated vaccine conjugated with alum adjuvant (DPIV, intramuscular, Day 0) and boosted with tetravalent dengue live attenuated virus (TDENV-LAV, subcutaneous, Day 28) followed by a challenge with dengue virus-2 (DENV-2, subcutaneous, Day 63). Day of the DENV-2 challenge was considered as the day of the last exposure for group one. Experimental groups two and three consisted of rhesus macaques that received a cell based, 293-gp96-Ig-PfCSP-PfAMA (gp96-Ig-PfCA) ( $\mathrm{n}=5, \mathrm{~F}=3, \mathrm{M}=2$ ) and NMRC-M3V-D/Ad-PfCSPPfAMA (D/Ad-PfCA) $(n=5, F=2, M=3)$ candidate malaria vaccines, respectively. The gp96-IgPfCA malaria vaccine had contained irradiated human embryonic kidney (HEK) 293 cells 
177

178

179

180

181

182

183

184

185

186

187

188

189

190

191

192

193

194

195

196

197

198

199

200

201

202

203

204

205

206

207

208

209

210

211

212

213

214

215

216

217

218

219

220

221

222

transfected with a mixture of plasmids encoding a secreted form of heat shock protein gp96 (gp96-Ig), Plasmodium falciparum circumsporozoite (PfCSP) protein and $P$. falciparum apical membrane antigen (PfAMA). The vaccine had been administered subcutaneously in three doses at 0,5 and 25 weeks. In D/Ad-PfCA heterologous prime-boost vaccination, macaques in the group three had been primed with three intramuscular doses containing a mixture of two DNA plasmids encoding PfCSP and PfAMA at 0, 4, 8 weeks and boosted once intramuscularly with a mixture of two non-replicating recombinant human serotype 5 adenovirus vectors expressing PfCSP and PfAMA antigens at 25 weeks. The day of last exposure for group two and three were therefore, 25 weeks post-last vaccination/boost.

\section{Sample Collection}

Whole blood was collected from the femoral vein directly into EDTA collection tubes for flow cytometry analysis or into serum separation tubes. Additional serum samples were obtained through tissue sharing agreements with the relevant dengue and malaria vaccine protocols. Whole blood samples $(100 \mu \mathrm{l})$ were processed within four hours of collection and stained cells were immediately acquired by multi-parametric flow cytometry for immunophenotyping. Serum samples $(75 \mu 1)$ were sub aliquoted into polypropylene micro tubes and stored at $-80^{\circ} \mathrm{C}$, and later used to determine the concentration of circulating cytokines and antigen-specific antibodies. Time points were determined based on availability of samples within each experimental group.

\section{Flow Cytometry}

Multi-parametric, flow cytometry was used to determine the phenotype and frequency of innate and adaptive immune cell subsets in the peripheral blood. Cell frequencies for experimental group one were measured at 1,2,3,4 and 6 months post-DENV challenge. Cell frequencies for experimental group two and three were measured at day 6, day 20, 2.5, 4, and 6 months post-last vaccination/boost. The nine color NHP immunophentyping panel was consisted of following surface antibodies from BD biosciences, San Jose, CA: CD45 (V450, clone D058-1283), CD3 (APC-Cy'TM, clone SP34-2), CD8 (APC, clone RPA-T8), CD16 (PE, clone 3G8), HLA-DR (BV605, clone G46-6); following antibodies from Miltenyi Biotec, San Diego, CA; CD20 (PEvio770, clone LT20), CD4 (Percp-Vio700, clone M-T466), CD159a (PE, clone REA 110), CD14 (FITC, clone TÜK4); and CD11c (Alexa Fluor 700, clone 3.9, Thermofisher scientific, Waltham, MA). Hundred microliters of whole blood sample was stained with a cocktail of flurochromeconjugated antibodies (seven color panel for group one and nine color panel for group two/three), incubated for $10 \mathrm{~min}$ at room temperature and diluted in sheath fluid for immediate acquisition. Acquisition was limited to cells expressing V450 fluorochrome/CD45 (trigger) at a particle cut-off size (FSC) of 4000 and 50,000 events/sample were acquired at a medium flow rate by 17-color, LSRII Fortessa flow cytometer at the ImmunoCore facility using the FACS DIVA software. Gating strategy used to analyze the phenotype and frequency of each immune cell subset is provided in the Figure S1. Flow data was analyzed by FCS express 6.0 software.

\footnotetext{
Absolute cell counts

Concentration of white blood cells in EDTA-preserved whole blood samples was determined using a Luna Dual Fluorescence Cell Counter within an hour of sample collection (Logos Biosystems, Annandale, VA). Ten microliters of whole blood was diluted in $990 \mu \mathrm{L}$ of 1x PBS (1:100), mixed by vortexing and $2 \mu \mathrm{L}$ of acridine orange/propodium iodide dye mix (Logos Biosystems, Annandale, VA) was added to $18 \mu \mathrm{L}$ of diluted blood sample. Ten microliter from
}

Peer) reviewing PDF | (2020:11:55408:1:1:REVIEW 22 Jan 2021) 
223

224

225

226

227

228

229

230

231

232

233

234

235

236

237

238

239

240

241

242

243

244

245

246

247

248

249

250

251

252

253

254

255

256

257

258

259

260

261

262

263

264

265

266

267

268

the mixture was loaded into Luna photon-slide disposable hemocytometer (VitaScientific, Beltsville, MD), and the concentration of fluorescent white blood cells was determined using the Luna dual fluorescence automated cell counter. Frequency of each individual cell subset determined by flow cytometry was used to calculate their absolute counts (cells $/ \mu \mathrm{L}$ ) as a proportion of the total white blood cells (CD45hi cells) (or thereby as a frequency of the parent cell population). Parent population of each immune cell subset described in the manuscript is shown in Figure S1.

\section{Cytokine/chemokine analysis by mesoscale assay}

A customized U-PLEX NHP biomarker panel of 12 cytokines/chemokines (IFN- $\gamma$, IL-1 $\beta$, IL-2, IL-4, IL-5, IL-6, IL-8, IL-10, IL-12/IL23p40, MCP-1, MIP-1 $\alpha$, TNF- $\alpha$ ) (Meso Scale discovery, Gaithersburg, MD) was used to analyze serum cytokine/chemokine levels per manufacture's instruction. All Cytokines for experimental group one were measured at day 7, 2 months, and 6 months, post-DENV-2 challenge. Cytokines for experimental group two and three were measured at day 6 , day 20, 2.5 months, and 6 months post-last vaccination/boost.

\section{Enzyme-linked immunosorbent assay (ELISA) for DENV antibodies}

ELISA assay was performed as described previously (Simmons, Sun \& Putnak, 2016) without any modifications.

\section{Statistical Analysis}

Data from the naïve group was collected independently from the three experimental groups (also data from the two malaria groups were collected independently from the DENV group).

Therefore, series of unpaired T-tests (two-tailed) were carried out to compare between the naïve group and each of the experimental groups at different time points post-exposure (alpha level of $0.05)$. Welch's correction was applied with unpaired $T$ test, when $P$ value of the $F$ test to compare variances were $\leq 0.05$. Data approximately conformed Shapiro-Wilk test and Kolmogorov-Smirnov tests for normality at 0.05 alpha level. Data were presented as mean \pm standard deviation in the text and in the figures. All statistical analysis were conducted using Graph Pad Prism 8 software. R studio (Version 1.2.1335) was used to generate correlograms (pearson correlation, two-tailed, 0.05 alpha level) and dendograms. Agglomerative hierarchical clustering with Pvclust was used to plot dendograms. AU P values were reported on the dendograms.

\section{Results}

\section{Innate immune cell dynamics in peripheral blood}

To study innate immune cell dynamics, we monitored frequencies and absolute cell counts of circulating monocytes (CD45+CD14+ or CD45+CD14+HLA-DR+), natural killer (NK) cells $(\mathrm{CD} 45+\mathrm{CD} 3-\mathrm{CD} 16 / \mathrm{CD} 159 \mathrm{a}+)$, NK cells expressing HLA-DR (CD45+CD3CD16/CD159a+HLA-DR+), natural killer T(NKT) cells (CD45+CD3+CD16/CD159a+), NKT cells expressing HLA-DR (CD45+CD3+CD16/CD159a+HLA-DR+) and dendritic cell (DC)s (CD45+CD14-CD16/CD159a-CD3-CD20-CD11c+HLA-DR+) in whole blood by flow cytometry and automated cell counting (Fig. S1 A, G and H). DC gating strategy is not shown in the figure, but described in the legend. 
269 Experimental group one consisted of rhesus macaques $(n=10)$ that had been primed with bivalent

270

271

272

273

274

275

276

277

278

279

280

281

282

283

284

285

286

287

288

289

290

291

292

293

294

295

296

297

298

299

300

301

302

303

304

305

306

307

308

309

310

311

312

313

314

or tetravalent dengue purified inactivated vaccine (DPIV) and boosted with tetravalent dengue live attenuated virus (TDENV-LAV) followed by a challenge with dengue virus-2 (DENV-2). Experimental group two had received three doses of a cell based malaria vaccine, containing irradiated HEK 293 cells secreting heat shock protein chaperon, gp96-Ig, and the two malaria antigens, circumsporozoite protein and apical membrane antigen-1 (gp96-Ig-PfCA) $(n=5)$. Experimental group three had been primed thrice with a mixture of DNA plasmids expressing the same two malaria antigens as in the experimental group two, and had been boosted once with non-replicating recombinant human serotype 5 adenovirus (Ad5) plasmids expressing the same antigens (D/Ad-PfCA) $(n=5)$. The pre-exposed animals were monitored at multiple time points, over 6 months since their most recent exposure (ex: last dose of vaccine or challenge). A group of naïve monkeys $(n=9)$ (age and sex matched, see materials and methods) were used as the controls.

All the NHP monocyte subsets explicitly express CD14 (Ziegler-Heitbrock, 2014), therefore, we used the same surface marker to identify circulating monocytes in rhesus macaques in the dengue vaccinated group (Fig. S1G). Since HLA-DR has been shown to reliably identify monocytes along with CD14 (Abeles et al., 2012), we later distinguished monocytes based on CD14 and HLA-DR co-expression for the two malaria vaccine groups (Fig. S1G). Due to some previous reports on lack of expression of CD56 on rhesus macaque NK cells (Carter et al., 1999; Shields et al., 2006), here we used CD159a, a previously validated marker for rhesus NK cells (Choi et al., 2008) in combination with CD16 (Fig. S1H).

Experimental group one, which, received prime-boost, DPIV/TDENV-LAV vaccine, showed elevated frequency of CD14+ monocytes at 1 month $(8.3 \pm 3.8, \mathrm{P}=0.007)$ and 3 months $(7.6 \pm 4.2$, $\mathrm{P}=0.03$ ) post $\mathrm{DENV}-2$ virus challenge compared to the naïve animals (3.9 \pm 1.8$)$. This transient expansion of monocytes were subsided to the levels present in the naïve macaques by 6 months post-challenge (Fig. 1A). While the absolute cell count data for this group is not available for early time points, we did not see any differences in the counts at late time points (4 and 6 months post-challenge) between vaccinated and naïve animals (data not shown). These data suggest that intermittent and transient alterations to circulating monocytes can be sustained up to 3 months post-DENV challenge. In contrast, no changes in the frequency and absolute cells counts of NK and NKT cell populations were observed (data not shown). For the group 1 macaques, the panel did not include surface markers for HLA-DR and DCs.

Dynamics of HLA-DR+CD14+ monocytes (both frequencies and cell counts) were found to be similar between rhesus macaques vaccinated with gp96-Ig-PfCA and naïve animals over the course of 6 months (Table S1). In contrast, animals vaccinated with D/Ad-PfCA showed a transient increase in monocyte frequencies at day 20 and at 4 months post-last boost compared to the naïve macaques (Fig. 1B, Table S1). We also noted that monocyte frequency of some of the individual macaques tend to stay consistently higher up to 4 months after their last exposure to Ad5 vectored malaria vaccine boost. Changes in circulating monocyte frequency did not impact the monocyte counts in D/Ad-PfCA exposed animals (Fig. 1C).

We did not observe any noticeable changes in NK cells, HLA-DR expressing NK cells, DC or NKT cells in the two malaria vaccine groups (data not shown). However, we noted more

PeerJ reviewing PDF | (2020:11:55408:1:1:REVIEW 22 Jan 2021) 
315

permanent alterations to a specific subset of NKT cells expressing HLA-DR activation marker (Fig. 2 and Table S1). NKT cells are known to share receptors expressed by both T and NK cells (Lanier, Spits \& Phillips, 1992). Previous evidence also suggests expression of HLA-DR on NKT cells under certain disease conditions or changes in cytokine milieu (Saikh \& Kissner, 2002; Almeida et al., 2019), which may play an important role in inflammation and immune regulation (Marrero, Ware, \& Kumar, 2015).

The frequency of HLA-DR expressing NKT cells were notably lower in most of the individual macaques pre-exposed to gp96-Ig-PfCA at 2.5 months and 4 months after the last vaccination (Fig. 2A and Table S1). HLA-DR+NKT cell counts were transiently reduced at 6 days post-last vaccination and returned to levels comparable to the naïve animals by 20 days. Thereafter, we observed more persistent reduction of HLA-DR+NKT cell numbers through 2.5 months, 4 months and 6 months post-exposure (Fig. 2B and Table S1).

In the D/Ad-PfCA malaria group, there was a persistent reduction of HLA-DR+NKT cell frequency through 2.5 months, 4 months and 6 months post-Ad5 boost compared to the naïve animals (Fig. 2C and Table S1). Subsequently, HLA-DR+NKT cell counts were persistently decreased through 2.5 months, 4 months and 6 months, followed by a transient decrease at 6 days post-Ad5 boost (Fig. 2D and Table S1). The data show that exposure to an adeno viralvector based, prime-boost malaria vaccination strategy could induce more consistent and permanent alterations to this rare NKT cell subset.

In summary, dynamic changes in circulating innate immune cell subsets appeared to be unique for their past exposures (ex: vaccination strategy or pathogen). Also, the data indicate that these changes can either be transient or more permanent, which could last up to 6 months.

\section{Circulating cytokines}

To determine changes in circulating cytokine milieu at multiple time points over 6 months postlast exposure, we used a customized, U-PLEX NHP biomarker panel of 12 cytokines (IFN-y, IL$1 \beta$, IL-2, IL-4, IL-5, IL-6, IL-8, IL-10, IL-12/IL23p40, MCP-1, MIP-1 $\alpha$, TNF- $\alpha$ ). Most of the cytokines were either non-detectable or present at levels less than $2 \mathrm{pg} / \mathrm{mL}$, except MCP-1, MIP$1 \alpha$ and IL-12/IL-23p40.

Monocyte chemoattractant protein-1/CCL2 (MCP-1) and macrophage inflammatory protein $\alpha /$ CCL3 (MIP1- $\alpha$ ) are two major chemokines (or a chemotactic cytokines) critical for leukocyte migration and infiltration (Deshmane et al., 2009; Bhavsar, Miller \& Al-Sabbagh, 2015). MIP1$\alpha /$ CCL3 plays an important role in modulating the inflammatory response, particularly in viral infections, by enhancing recruitment of leukocytes (ex. macrophages, and lymphocytes) to the site of inflammation (Bhavsar, Miller \& Al-Sabbagh, 2015). We found slightly increased levels of MCP-1 in some of the individual animals exposed to DENV-2 challenge $(210.3 \pm 105.5$, $\mathrm{P}=0.059)$ by 6 months compared to naïve animals (133.9 \pm 47.1$)$ (Fig. 3A). In contrast, most of the animals had persistently decreased MIP1- $\alpha$ levels, 7 days through 2 months post-DENV-2 virus challenge, but failed to reach the statistical significance (Fig. 3A). Both MCP-1 and MIP1$\alpha$ levels in the two malaria vaccine groups were comparable to naïve animals throughout the 6 month follow-up period (Fig. 3B and C). 
361 In fact, compared to MCP-1 and MIP1- $\alpha$ dynamics, more pronounced persistent alterations were 362 noted in circulating concentrations of interleukin (IL)-12/IL23p40 cytokines in pre-exposed macaques. IL-12 and IL-23 are heterodimers that share the p40 subunit, mainly produced by dendritic cells and macrophages. IL-12 mainly regulates differentiation of T helper 1 (Th1) cells and interferon gamma (IFN- $\gamma$ ) cytokine production. IL-23 is crucial for maintenance and expansion of Th17 cells (Lyakh et al., 2008). IL-12/IL-23p40 cytokines were persistently lower in DPIV/TDENV-LAV vaccinated animals at 7 days (47.9 $\pm 14.1, \mathrm{P}=0.007), 2$ months $(59.9 \pm 18.3, \mathrm{P}=0.01)$ and 6 months $(65.7 \pm 22.9, \mathrm{P}=0.03)$ post-DENV-2 challenge, than the naïve animals (115.7 \pm 52.3$)$ (Fig. 3A). Slower but gradual increase of mean values post-DENV-2 challenge, indicates that altered IL-12/IL-23p40 levels may be returning back to its steady state over the time (Fig. 3A). We observed a similar trend in D/Ad-PfCA group, with more transient reduction of IL-12/IL-23p40 at 6 days $(59.0 \pm 4.6, \mathrm{P}=0.01)$ and 20 days $(65.6 \pm 18.7, \mathrm{P}=0.03)$ postAd5 boost (Fig. 3C). This was followed by a gradual increase at 2.5 months $(73.9 \pm 21.2, \mathrm{P}=0.12)$, subsequently returning to the levels found in the naïve group $(115.7 \pm 52.3)$ by 6 months $(120.7 \pm$ 41.6, $\mathrm{P}=0.8$ ) (Fig. 3C). Rhesus macaques vaccinated with gp96-Ig-PfCA had lower mean concentrations of IL-12/IL-23p40, increasing over time post-last vaccination, yet remained statistically comparable to the naïve animals (Fig. 3B).

Dynamics of IL-12/IL-23p40 seemed to follow a trend unique to each exposure, when shifting from altered state to the steady or naïve state. This transition may take up to 2.5 months or longer than 6 months post-last exposure, depending on the type of the exposure. Our findings agree with the fact that different exposures can induce either transient or persistent changes, uniquely altering the dynamics and composition of the circulating cytokine milieu.

\section{B cells}

In order to evaluate global impact on the humoral arm of the adaptive immunity, we determined the cell frequency and counts of circulating B cells. Interestingly, a persistent reduction in the overall CD20+B cell frequency was seen after DENV-2 challenge (Fig. 4A and Table S2). Although data are not available for the first three time points (1,2 and 3 months), B cell counts at 4 months and 6 months post-challenge were still significantly lower than the naïve group (Fig. 4B and Table S2). It appears that the majority of circulating B cells may have been either suppressed or have migrated to the secondary lymphoid organs, presumably as a result of the prime-boost DPIV/TDENV-LAV vaccination. High titers of antibodies against all the four dengue serotypes (1-4) were detected at 2 months and 6 months post-DENV-2 challenge (Fig. S2A). The ability of DPIV/TDENV-LAV vaccine strategy to elicit high levels of DENV 1-4 specific antibodies may likely be associated with the dynamics of circulating B cells.

As shown in Figure 4C, the D/Ad-PfCA group also had decreased B cell frequencies at 6 days and 20 days, which then became comparable to the naïve animals by 2.5 months post-Ad5 boost. Our data suggest that this transient stabilization after the acute phase of peripheral immune response is followed by a second wave of more persistent alterations, as the D/Ad-PfCA group showed persistently reduced B cell frequencies at 4 months and 6 months (Fig 4C and Table S2). Variations in the B cell frequencies in D/Ad-PfCA vaccinated animals subsequently affected the B cell counts up to 4 months post-Ad5 boost. Nevertheless, by 6 months, B cell counts of D/AdPfCA group were comparable to the naïve animals, despite the differences observed in B cell frequencies (Fig. 4D and Table S2). In contrast to the D/Ad-PfCA group and DENV group, B 
407

408

409

410

411

412

413

414

415

416

417

418

419

420

421

422

423

424

425

426

427

428

429

430

431

432

433

434

435

436

437

438

439

440

441

442

443

444

445

446

447

448

449

450

451

cell frequencies and counts in gp96-Ig-PfCA vaccinated animals were maintained within the range found in the naïve animals (Fig. 4C, 4D and Table S2).

Based on the differential ability of DPIV/TDENV-LAV, D/Ad-PfCA and gp96-Ig-PfCA vaccination strategies to elicit antibodies (Fig. S2A and S2B), it may be reasonable to speculate that there is a long-term interplay between circulating B cells and antigen-specific antibody production. As we have seen with the other components of the immunity in the present study, changes in global peripheral B cell population also appeared to be exposure-specific.

\section{T cells}

T cells are the central players of cell mediated immunity, which can modulate the function of many immune cells, activate host defense mechanisms and lyse pathogen infected cells. To understand global changes in circulating T cells following exposure to different protocols, we determined the frequency and cell counts of various $\mathrm{T}$ cell subsets. CD3+ T cell subsets were analyzed on $\mathrm{CD} 3+$ gated population (Fig. S1C-F).

We found decreased CD3 + gated CD8 + T cell frequencies in DPIV/TDENV-LAV vaccinated animals, up to 1 month DENV-2 post-challenge $(15.6 \pm 8.7, \mathrm{P}=0.003)$, compared to the naïve group (26.6 \pm 2.0$)$ (Fig. 5A). This contraction appeared to be associated with the expansion of double negative (DN) T cells (vaccinated group-23.9 $\pm 6.3 \mathrm{p}=0.0002$; Naïve-11.9 \pm 4.0 ) (Fig. 5A). Frequencies of CD8+ T cells and DN cells were returned to the naïve levels after 1 month. Notably, frequency of CD3 + T cells and CD3 + gated CD4+ T cells remained unchanged throughout the follow-up (data not shown). Also, the $\mathrm{T}$ cell counts obtained for each cell subset at 4 months and 6 months for the vaccinated group did not differ from the naïve animals (data not shown) (data is not available for the first three months).

Gp96-Ig-PfCA vaccination did not induce any alterations to the circulating $\mathrm{T}$ cells, including CD3 $+\mathrm{T}$ cells, CD3 + gated CD4+ T cells and CD8+ T cells (data not shown). Figure 5B and 5C show that both cell frequency $(53.7 \pm 4.1, \mathrm{P}=0.02)$ and the cell counts $(1753 \pm 803, \mathrm{P}=0.04)$ of CD3+ gated CD4+ T cell population were transiently decreased by day 20 post-Ad5 boost, subsequently returning to the levels seen in naïve animals (Frequency $60.4 \pm 5.0$, counts$3808 \pm 1958$ ) by 2.5 months. Nevertheless, CD4+ T cell counts seemed to continuously increase over 6 months post-Ad5 boost (within the range seen in naive animals), rather than remaining in a steady state (Fig. 5C). CD3+ T cells and CD3+ gated CD8+ T cell populations were not affected by D/Ad-PfCA vaccination (data not shown).

We then analyzed the dynamics of CD3 + T cell subsets expressing HLA-DR, a well-known marker for activated T cells (Shipkova \& Wieland, 2012). We observed a gradual reduction of frequency and cell counts of activated T cells (CD3+HLA-DR+), CD8+ T cells (CD3+ gated CD8+HLA-DR+) and CD4+ T cells (CD3+ gated CD4+HLA-DR+), over 6 months post-last gp96-Ig-PfCA vaccination compared to naïve animals (Fig. 6A, 6B and Table S3). Impact of vaccination was more profound on frequency and cell count of activated CD8+ T cells, than on the activated CD4+ T cell population, from 2.5 months through 6 months post-last boost (Fig. 6A, 6B and Table S3). Long-term alterations to activated T cell subsets, particularly the CD8+ T cells, may likely have been associated with the ability of gp96-Ig based vaccines to activate 
452

453

454

455

456

457

458

459

460

461

462

463

464

465

466

467

468

469

470

471

472

473

474

475

476

477

478

479

480

481

482

483

484

485

486

487

488

489

490

491

492

493

494

495

496

497

cellular arm of the immunity and to induce CD8+ T cell mediated tissue-resident memory (Strbo et al., 2011; Strbo et al., 2016; Strbo et al., 2020).

As shown in Figure 6C, after Ad5 boost, we found elevated frequency of activated CD3+ T cells by 20 days, followed by a gradual decrease to naïve levels (Table S4). In contrast, activated CD4+ T cells (CD3+ gated) maintained higher mean frequencies than naïve animals throughout the 6-month follow-up period, where the values were statistically significant at 20 days and 2.5 months (Fig. 6D and Table S4). Changes in frequencies of activated CD3+ T cells and CD3+ gated CD4+ T cells did not alter their cell counts (Table S4). Dynamics of activated CD8+ T cells remained comparable to naïve animals (Table S4). Our data indicate that the D/Ad-PfCA malaria vaccine mainly alter the $\mathrm{CD} 4+\mathrm{T}$ cell component in circulation, inducing persistent changes over 6 months post-last exposure.

Here we show that each individual exposure could alter the global balance of cell mediated adaptive immune responses in the peripheral blood, irrespective of the anticipated vaccinefocused, antigen-specific, cell-mediated immune responses.

\section{Global immune profile}

We next sought to determine whether the observed changes in individual immune cell subsets could subsequently affect the overall immune profile of pre-exposed rhesus macaques (Fig. 7). We used correlation matrices and hierarchical clustering to visualize the relationships between cell counts of different cell types within Naïve (Fig. 7A), gp96-Ig-PfCA (Fig. 7B) and D/AdPfCA (Fig. 7C) groups. Immune system consists of a highly integrated network of immune cell subsets, where changes in cell counts of a certain immune cell subset could be positively or negatively related to the counts of another immune cell type, particularly after exposing to different antigenic stimuli. For example, counts of certain circulating immune cell subsets could be synergistically altered due to leukocyte migration during an inflammatory immune response. These associations subsequently represent the overall immune profile of an individual.

Correlation coefficient of cell counts was computed for all possible combinations (pairs) of immune cell subsets within each group to generate a correlation matrix and then was visualized by a correlation plot (correlogram) using R studio software (see methods-statistical analysis). In other words, each correlogram represents negative and positive relationships between the cell counts of all pairs of immune cell subsets tested within each group (Fig. 7). Correlograms of the two vaccinated groups, gp96-Ig-PfCA (Fig. 7B) and D/Ad-PfCA (Fig. 7C) displayed a remarkable deviation from the naïve group (Fig. 7A). Dendrograms were generated using hierarchical clustering to achieve a higher resolution view of these relationships. Here, the cell clusters represent closely correlating measurements where the length of the clades represents the extent of similarity and closeness between these measurements (Fig. 7, dendograms). Both naïve animals (Fig. 7A) and the D/Ad-PfCA group (Fig. 7C) had two major immune cell clusters (AU $=100$, highlighted by a red rectangle). The two groups shared one of these major clusters containing NKT-NK cell clade $(A U=100)$. However, the three $\mathrm{T}$ cell clades in the second cluster showed distinctive differences between the two groups. More interestingly, the gp96-Ig-PfCA group had 5 distinctive clades, with only one strongly associated CD8+ T cytotoxic-NKT cells cell cluster ( $\mathrm{AU}=100$, highlighted by red rectangle) (Fig. 7B). Differentially associated immune cell clusters, unique to each group, indicate a distinctive interplay among immune cell 
498

499

500

501

502

503

504

505

506

507

508

509

510

511

512

513

514

515

516

517

518

519

520

521

522

523

524

525

526

527

528

529

530

531

532

533

534

535

536

537

538

539

540

541

542

components based on their antigenic exposure. Thus, our data collectively suggest that each group could develop a unique global immune profile in peripheral blood under different experimental conditions.

\section{Discussion}

Repeated exposure to various physical and mental stimuli could modulate overall biology of NHPs, with possible implications on re-using those animals between various research protocols. However, so far, we have lacked proper scientific evidence to justify the widely accepted, arbitrarily implemented, 4-6 weeks rest-period between re-assignment of NHPs in different research protocols. One way to understand the impact on overall biology due to a certain exposure is to study dynamics of key biological systems of the exposed NHPs. Changes in the immune system in fact, are better indicators of overall biological impact, due to its continuous cross-talk with the rest of the body compartments (Zmora et al., 2017; Limanaqi et al., 2019; Poggi et al., 2019; Singbartl, Formeck \& Kellum, 2019). Here, we show that exposure of rhesus macaques to various antigenic stimuli could largely alter overall dynamics of the immune system in peripheral blood. The alterations were unique to each exposure. While some of the alterations were transient, some changes lasted for more than six months, emphasizing the need for closer and case-by-case analysis of animal's overall immunity, before re-assigning them to new protocols.

An increasing body of evidence suggests that exposure to various environmental insults could induce epigenetic modifications leading to sustainable changes in transcriptional programming of cells (Hamada et al., 2019). This in fact, is believed to alter the functional state of certain innate immune cells, such as monocytes and cytokines, which is sustained for weeks or months after eliminating the initial antigenic or microbial stimulus (Netea et al., 2016; Hamada et al., 2019; Zhang \& Cao, 2019). Previous studies have demonstrated long-term functional reprogramming of myeloid cells following vaccinations and acute viral infections (Kleinnijenhuis et al., 2012; Yao et al., 2018; Aegerter et al., 2020). A similar phenomenon would presumably explain the alterations seen in dynamics of circulating monocytes and cytokines that occurred over 6 months following DENV-2 challenge and Ad5 boost (Fig. 1 and Fig. 3). For example, DPIV/TDENVLAV vaccination and/or DENV-2 challenge could reprogram the transcription of circulating MIP1- $\alpha$ and IL-12/IL-23p40 cytokines, leading to a long-term suppression of their secretion (Fig. 3A). MIP-1 $\alpha$ has been shown to play a role in DENV immunopathology (Spain-Santana et al., 2001), therefore, observed suppression of MIP-1 $\alpha$, would presumably be related to preventing progression of dengue viral infection in vaccinated animals. Likewise, IL-12/IL23p40 suppression seen following DENV-2 challenge may likely have been associated with cross-interference between RIG-I-like receptor (RLR) and Toll-like receptor (TLR) signaling pathways in innate immune cells (Negishi et al., 2012; Sprokholt, Helgers \& Geijtenbeek, 2017). Although, IL-12 is crucial for Th1 differentiation to fight intracellular pathogens such as viruses (Komastu, Ireland \& Reiss, 1998), only IFN- $\alpha$ and IL-27 appear to mediate Th1 polarization following DENV infections (Sprokholt et al., 2017a; Sprokholt et al., 2017b). Nevertheless, the functional relevance of the observed changes in terms of development of innate immunological memory leading to "trained immunity" or "tolerance" (Rodriguez, Suarez-Alvarez, \& LopezLarrea, 2019) has yet to be elucidated. Non-specific heterologous effects of some of the widelyused live attenuated vaccines such as BCG and measles are now thought to be mediated by 
543 innate immune memory (Arts et al., 2016). Similar effects would lead to false conclusions, even 544 if the NHPs are being re-used in unrelated pre-clinical vaccine studies.

545

546

547

548

549

550

551

552

553

554

555

556

557

558

559

560

561

562

563

564

565

566

567

568

569

570

571

572

573

574

575

576

577

578

579

580

581

582

583

584

585

586

587

588
Besides all the non-specific, innate immune effects, exposure to antigens or microbes always unfolds a series of antigen-specific immunological effects shaping adaptive immunity. Indeed, as we observed, these antigen-specific effects seem to overtly dominate the steady-state of B cell dynamics, lasting for months, more profoundly after DPIV/TDENV-LAV-DENV-2 and to some extent after DNA/Ad-PfCA exposures (Fig. 4). We also believe that persistent reductions observed in B cells more likely have been associated with maintenance of antigen-specific antibodies in the two vaccinated groups. In fact, short-lived, anti-PfCSP antibodies were produced in smaller quantities relative to more persistent, larger quantities of anti-DENV antibodies (Fig. S2), which might explain the more profound decrease of B cells in the dengue vaccinated group (Fig. 4A and 4B). Theoretically, putative antigen depots displayed in follicular dendritic cells could allure naïve B cells from the circulation contributing to the observed dynamics (Heesters et al., 2016; Kranich \& Krautler, 2016). It is also plausible that memory B cells from the circulating pool may continuously be recruited to replenish the pool of plasma cells (Traggiai, Puzone \& Lanzavecchia, 2003). While the exact reasons for these altered B cell dynamics are unknown, we believe that as with $\mathrm{B}$ cells, alterations seen in the $\mathrm{T}$ cell dynamics may have been also pre-programmed by the particular vaccination strategy.

More intriguingly, the fact that the different antigenic stimuli could generate unique patterns of associations between various immune cell subsets (Fig. 7) further validates our hypothesis of exposure-specific, global immune imprinting in rhesus macaques. In this modified immunological state, repeated use of pre-exposed animals may affect consistency and reproducibility of subsequent research data. Instead, our findings necessitate the development of more detailed screening criteria for re-used NHPs prior to enrollment in any subsequent protocol. The 9-color flow cytometry panel integrated with 12 U-Plex NHP cytokine panel used in this study provide a basic tool for immune-profiling. Antibody panel could be customized to detect additional immune cell subsets such as memory and regulatory cells based on the experimental needs or user preference. We recommend analysis of fresh whole blood samples, instead of purified peripheral blood mononuclear cells (PBMC), to avoid technical bias generated by purification, freeze-thawing of cells, etc. Our data warrant regular collection of blood and serum samples at baseline and at several time-points post-last exposure to evaluate, long-term dynamics of immune cells and cytokines, particularly after major immunological stressors, whether they be resultant from experimentation, social dynamics, or transportation stress. More specifically, our findings lay the groundwork for further investigations when considering re-use of pre-exposed rhesus macaques in infectious disease research. However, the present study was limited to groups of rhesus macaques housed at the WRAIR animal facility, which have been exposed to three experimental vaccines, and only one group was challenged with a live pathogen. Further research is required to determine the repeatability of these findings, particularly after exposure to other infectious agents, vaccinations, and novel therapeutics. Exposure to live pathogens would undoubtedly lead to more robust immune responses, which may result in immediate and prolonged changes in the immune cell dynamics (Silveira et al., 2017). Immunological changes observed in pre-exposed NHPs may also vary by the animal facility, NHP colonies, and the NHP species. Each animal facility has different environmental stressors/stimuli, where certain containments or facilities are maintained under germ-free conditions. Genetic variations in major 
589 histocompatibility complex exist among different NHP species or sub-populations (Viray, Rolfs

590 \& Smith, 2001; Heijmans, de Groot \& Bontrop, 2020). This would potentially generate species

591 or population-specific immune responses even when exposed to the same

592 antigenic/environmental stimuli. Therefore, aforementioned variables should be carefully

593 considered when developing flow-cytometry antibody panels or cytokine panels to screen re-

594 used NHPs. The strategy of immune-profiling NHPs prior to incorporating them into

595 experimental studies would have two interrelated benefits - more consistent experimental results

596 by randomly re-assigning pre-exposed animals into new studies to avoid study bias and

597 improved adherence to the 3Rs of research. Use of NHPs with less immunological variations,

598 thereby presumably less overall biological variations, would provide better pre-clinical models in

599 biomedical research. This, in turn, would allow the use of fewer NHPs and maximize the

600 information obtained from them consistent with Animal Welfare Act and the reduction principle

601 of the 3 Rs.

602

603

\section{Conclusions}

604 Every different antigenic exposure or environmental stimulus can generate a unique global 605 immune profile in NHPs, potentially impacting their overall biology and subsequent re-use in 606 research experiments. To test this hypothesis we studied dynamics of circulating immune cell 607 subsets (innate and adaptive) and cytokines in rhesus macaques previously exposed to different 608 antigenic stimuli. We observed exposure-specific, transient and persistent changes (up to 6 609 months), in different immune cell populations as well as in the cytokine profile. Our data suggest 610 that overall biological changes represented by exposure-related, global immune imprinting 611 should be carefully evaluated before re-assigning NHPs to new studies to avoid study bias. 612 Further research is warranted to understand whether such changes could lead to "trained613 immunity" or "immune tolerance" and subsequent impact of NHP re-use on the experimental 614 outcome. 


\section{Acknowledgements}

616 We sincerely thank Ms Dawn Wolf, Ms Marcia Caputo and all the staff at Veterinary Services

617 Program, Walter Reed Army Institute of Research, for their extensive support with non-human

618 primate work reported in this article. We also thank Dr Martha Sedegah, Ms Noelle Patterson

619 and members of the Malaria Department, Naval Medical Research Center for sharing samples,

620 Dr Gregory Gromowski at Viral Diseases Branch, Walter Reed Army Institute of Research for

621 sharing samples and background information on the dengue vaccination strategy reported in this

622 article. FB is a military service member and ED, MS, EV and EBL are US Government

623 employees. The views expressed in this article are those of the authors and do not necessarily

624 reflect the official policy or position of the Department of the Navy, Department of Defense, nor

625 the U.S. Government. Material has been reviewed by the Walter Reed Army Institute of

626 Research. There is no objection to its presentation and/or publication. The opinions or assertions

627 contained herein are the private views of the author, and are not to be construed as official, or as

628 reflecting true views of the Department of the Army or the Department of Defense. Also, the

629 views expressed in this article do not necessarily reflect the official policy or position of The

630 Henry M. Jackson Foundation for the Advancement of Military Medicine, Inc. (HJF). For

631 military service members or employees of the U.S. Government this work was prepared as part

632 of their official duties. Title 17 U.S.C. $\S 105$ provides that 'Copyright protection under this title is

633 not available for any work of the United States Government.' Title 17 U.S.C. $\$ 101$ defines a U.S.

634 Government work as a work prepared by a military service member or employee of the U.S.

635 Government as part of that person's official duties.

636

637

638

639

640

641

642

643

644

645

646

647

648

649

650

651

652

653

654

655

656

657

658

\section{References}

Abeles RD, McPhail MJ, Sowter D, Antoniades CG, Vergis N, Vijay GK, Xystrakis E, Khamri W, Shawcross DL, Ma Y, Wendon JA, Vergani D. 2012. CD14, CD16 and HLA-DR reliably identifies human monocytes and their subsets in the context of pathologically reduced HLA-DR expression by CD14(hi)/CD16(neg) monocytes: Expansion of CD14(hi)/CD16(pos) and contraction of CD14(lo) /CD16(pos) monocytes in acute liver failure. Cytometry 81(10): 823834

Aegerter H, Kulikauskaite J, Crotta S, Patel H, Kelly G, Hessel EM, Mack M, Beinke S, Wack A. 2020. Influenza-induced monocyte-derived alveolar macrophages confer prolonged antibacterial protection. Nature immunology 21(2):145-157

Almeida JS, Couceiro P, López-Sejas N, Alves V, Růžičková L, Tarazona R, Solana R, FreitasTavares P, Santos-Rosa M, Rodrigues-Santos P. 2019. NKT-Like (CD3+CD56+) Cells in Chronic Myeloid Leukemia Patients Treated With Tyrosine Kinase Inhibitors. Frontiers in immunology 10: 2493; 10.3389/fimmu.2019.02493

Arts RJW, Carvalho A, La Rocca C, Palma C, Rodrigues F, Silvestre R, Kleinnijenhuis J, Lachmandas E, Gonçalves LG, Belinha A, Cunha C, Oosting M, Joosten LAB, Matarese G, van Crevel R, Netea MG. 2016. Immunometabolic Pathways in BCG-Induced Trained Immunity. Cell Reports 17(10): 2562-2571 
659 Bell JD, Bergin IL, Schmidt K, Zochowski MK, Aronoff DM, Patton DL. 2011. Nonhuman 660 primate models used to study pelvic inflammatory disease caused by Chlamydia trachomatis.

Benn CS, Netea MG, Selin LK, Aaby PA. 2013. small jab - a big effect: nonspecific immunomodulation by vaccines. Trends in Immunology 34(9): 431-439

Bhavsar I, Miller CS, Al-Sabbagh M. Macrophage Inflammatory Protein-1 Alpha (MIP-1 alpha)/CCL3: As a Biomarker. General Methods in Biomarker Research and their Applications, Biomarkers in Disease: Methods, Discoveries and Applications (eds. Preedy VR, Patel VB.) 223-249 (Springer Science+Business Media Dordrecht 2015).

Blair TC, Manoharan M, Rawlings-Rhea SD, Tagge I, Kohama SG, Hollister-Smith J, Ferguson B, Woltjer RL, Frederick MC, Pollaro J, Rooney WD, Sherman LS, Bourdette DN, Wong SW. 2016. Immunopathology of Japanese macaque encephalomyelitis is similar to multiple sclerosis. Journal of Neuroimmunology 291: 1-10

Blok BA, Arts RJ, van Crevel R, Benn CS, Netea MG. 2015. Trained innate immunity as underlying mechanism for the long-term, nonspecific effects of vaccines. Journal of Leukocyte Biology 98(3): 347-356

Blok BA, de Bree LCJ, Diavatopoulos DA, Langereis JD, Joosten LAB, Aaby P, van Crevel R, Benn CS, Netea MG. 2020. Interacting, Nonspecific, Immunological Effects of Bacille CalmetteGuérin and Tetanus-diphtheria-pertussis Inactivated Polio Vaccinations: An Explorative, Randomized Trial. Clinical Infectious Diseases 70(3): 455-463

Calapai A, Berger M, Niessing M, Heisig K, Brockhausen R, Treue S, Gail A. 2017. A cagebased training, cognitive testing and enrichment system optimized for rhesus macaques in neuroscience research. Behavior Research Methods 49(1): 35-45

Capitanio JP, Cole SW. 2015. Social instability and immunity in rhesus monkeys: the role of the sympathetic nervous system. Philosophical transactions of the Royal Society of London. Series B, Biological sciences 370(1669): 20140104; 10.1098/rstb.2014.0104

Carlsson HE, Schapiro SJ, Farah I, Hau J. 2004. Use of primates in research: a global overview. American journal of primatology 63(4): 225-237

Carter DL, Shieh TM, Blosser RL, Chadwick KR, Margolick JB, Hildreth JE, Clements JE, Zink MC. 1999. CD56 identifies monocytes and not natural killer cells in rhesus macaques. Cytometry 37(1): $41-50$

Choi EI, Wang R, Peterson L, Letvin NL, Reimann KA. 2008. Use of an anti-CD16 antibody for in vivo depletion of natural killer cells in rhesus macaques. Immunology 124(2): 215-222 
703

704

705

706

707

708

709

710

711

712

713

714

715

716

717

718

719

720

721

722

723

724

725

726

727

728

729

730

731

732

733

734

735

736

737

738

739

740

741

742

743

744

745

746

747

748

de Bree LCJ, Koeken VACM, Joosten LAB, Aaby P, Benn CS, van Crevel R, Netea MG. 2018. Non-specific effects of vaccines: Current evidence and potential implications. Seminars in Immunology 39: 35-43

Deshmane SL, Kremlev S, Amini S, Sawaya BE. 2009. Monocyte chemoattractant protein-1 (MCP-1): an overview. Journal of Interferon \& Cytokine Research 29(6): 313-326

Didier ES, Sugimoto C, Bowers LC, Khan IA, Kuroda MJ. 2012. Immune correlates of aging in outdoor-housed captive rhesus macaques (Macaca mulatta). Immunity \& Ageing 9(1): 25; $10.1186 / 1742-4933-9-25$

Epstein MM, Vermeire T. 2017. An Opinion on non-human primates testing in Europe. Drug Discovery Today Disease Models 23: 5-9

Hamada A, Torre C, Drancourt M, Ghigo E. 2019. Trained Immunity Carried by Non-immune Cells. Frontiers in microbiology 9: 3225; 10.3389/fmicb.2018.03225

Heesters BA, van der Poel CE, Das A, Carroll MC. 2016. Antigen Presentation to B Cells. Trends in Immunology. 37(12): 844-854

Heijmans CMC, de Groot NG, Bontrop RE. 2020. Comparative genetics of the major histocompatibility complex in humans and nonhuman primates. International Journal of Immunogenetics 47(3):243-260

Kandasamy R, Voysey M, McQuaid F, de Nie K, Ryan R, Orr O, Uhlig U, Sande C, O'Connor D, Pollard AJ. 2016. Non-specific immunological effects of selected routine childhood immunisations: systematic review. BMJ 355: i5225; 10.1136/bmj.i5225

Kleinnijenhuis J, Quintin J, Preijers F, Joosten LA, Ifrim DC, Saeed S, Jacobs C, van Loenhout J, de Jong D, Stunnenberg HG, Xavier RJ, van der Meer JW, van Crevel R, Netea MG. 2012. Bacille Calmette-Guerin induces NOD2-dependent nonspecific protection from reinfection via epigenetic reprogramming of monocytes. Proceedings of the National Academy of Sciences $U S$ A 109(43): 17537-17542

Komastu T, Ireland DD, Reiss CS. 1998. IL-12 and viral infections. Cytokine \& Growth Factor Reviews. 9(3-4): 277-285

Kranich J, Krautler NJ. 2016. How Follicular Dendritic Cells Shape the B-Cell Antigenome. Frontiers in Immunology 7: 225; 10.3389/fimmu.2016.00225

Lanier LL, Spits H, Phillips JH. 1992. The developmental relationship between NK cells and T cells. Immunology Today 13(10): 392-395

Lankau EW, Turner PV, Mullan RJ, Galland GG. 2014. Use of nonhuman primates in research in North America. Journal of the American Association for Laboratory Animal Science 53(3): 278-282 
749

750

751

752

753

754

755

756

757

758

759

760

761

762

763

764

765

766

767

768

769

770

771

772

773

774

775

776

777

778

779

780

781

782

783

784

785

786

787

788

789

790

791

792

793

Limanaqi F, Biagioni F, Gaglione A, Busceti CL, Fornai F. 2019. A Sentinel in the Crosstalk Between the Nervous and Immune System: The (Immuno)-Proteasome. Frontiers in

Immunology 10: 628; 10.3389/fimmu.2019.00628

Lyakh L, Trinchieri G, Provezza L, Carra G, Gerosa F. 2008. Regulation of interleukin12/interleukin-23 production and the T-helper 17 response in humans. Immunological Reviews 226: $112-131$

Marrero I, Ware R, Kumar V. 2015. Type II NKT Cells in Inflammation, Autoimmunity, Microbial Immunity, and Cancer. Frontiers in Immunology 6: 316; 10.3389/fimmu.2015.00316

Nehete PN, Shelton KA, Nehete BP, Chitta S, Williams LE, Schapiro SJ, Abee CR. 2017. Effects of transportation, relocation, and acclimation on phenotypes and functional characteristics of peripheral blood lymphocytes in rhesus monkeys (Macaca mulatta). PLoS One. 12(12): e0188694; 10.1371/journal.pone.0188694

Negishi H, Yanai H, Nakajima A, Koshiba R, Atarashi K, Matsuda A, Matsuki K, Miki S, Doi T, Aderem A, Nishio J, Smale ST, Honda K, Taniguchi T. 2012. Cross-interference of RLR and TLR signaling pathways modulates antibacterial T cell responses. Nature Immunology 13(7): 659-666

Netea MG, Joosten LA, Latz E, Mills KH, Natoli G, Stunnenberg HG, O'Neill LA, Xavier RJ. 2016. Trained immunity: A program of innate immune memory in health and disease. Science. 352: 6284, aaf1098; 10.1126/science.aaf1098

Netea MG, Giamarellos-Bourboulis EJ, Domínguez-Andrés J, Curtis N, van Crevel R, van de Veerdonk FL, Bonten M. 2020. Trained immunity: a tool for reducing susceptibility and severity of SARS-CoV-2 infection. Cell. https://doi.org/10.1016/j.cell.2020.04.042

Poggi A, Benelli R, Venè R, Costa D, Ferrari N, Tosetti F, Zocchi MR. 2019. Human GutAssociated Natural Killer Cells in Health and Disease. Frontiers in Immunology 10: 961; 10.3389/fimmu.2019.00961

Prescott MJ, Bowell VA, Buchanan-Smith HM. 2004. Training laboratory-housed non-human primates, part 2: Resources for developing and implementing training programmes. Animal Technology \& Welfare 4: 133-148

Raijmakers RP, Stenos J, Keijmel SP, Ter Horst R, Novakovic B, Nguyen C, Van Der Meer JW, Netea MG, Bleeker-Rovers CP, Joosten LA, Graves SR. 2019. Long-Lasting Transcriptional Changes in Circulating Monocytes of Acute Q Fever Patients. Open Forum Infectious Diseases 6(7): ofz296; doi:10.1093/ofid/ofz296

Raper J, Bosinger S, Johnson Z, Tharp G, Moran SP, Chan AWS. 2016. Increased irritability, anxiety, and immune reactivity in transgenic Huntington's disease monkeys. Brain, Behavior, \& Immunity 58: 181-190 
794

795

796

797

798

799

800

801

802

803

804

805

806

807

808

809

810

811

812

813

814

815

816

817

818

819

820

821

822

823

824

825

826

827

828

829

830

831

832

833

834

835

836

837

838

839

Rodriguez RM, Suarez-Alvarez B, Lopez-Larrea C. 2019. Therapeutic Epigenetic

Reprogramming of Trained Immunity in Myeloid Cells. Trends in Immunology 40(1): 66-80

Saikh KU, Kissner T, Ulrich RG. 2002. Regulation of HLA-DR and co-stimulatory molecule expression on natural killer $\mathrm{T}$ cells by granulocyte-macrophage colony-stimulating factor. Immunology. 106(3): 363-372

Scott L, Pearce P, Fairhall S, Muggleton N, Smith J. 2003. Training nonhuman primates to cooperate with scientific procedures in applied biomedical research. Journal of Applied Animal Welfare Science 6(3): 199-207

Shields LE, Sieverkropp AJ, Potter J, Andrews RG. 2006. Phenotypic and cytolytic activity of Macaca nemestrina natural killer cells isolated from blood and expanded in vitro. American Journal of Primatology 68(8): 753-764

Shipkova M, Wieland E. 2012. Surface markers of lymphocyte activation and markers of cell proliferation. Clinica chimica acta; international journal of clinical chemistry 413(17-18): $1338-1349$

Silveira ELV, Rogers KA, Gumber S, Amancha P, Xiao P, Woollard SM, Byrareddy SN, Teixeira MM, Villinger F. 2017. Immune Cell Dynamics in Rhesus Macaques Infected with a Brazilian Strain of Zika Virus. Journal of Immunology 199(3): 1003-1011

Simmons M, Sun P, Putnak R. 2016. Recombinant Dengue 2 Virus NS3 Helicase Protein Enhances Antibody and T-Cell Response of Purified Inactivated Vaccine. PLoS One. 11(4):e0152811.

Singbartl K, Formeck CL, Kellum JA. 2019. Kidney-Immune System Crosstalk in AKI. Seminars in nephrology 39(1): 96-106

Spain-Santana TA, Marglin S, Ennis FA, Rothman AL. 2001. MIP-1 alpha and MIP-1 beta induction by dengue virus. Journal of Medical Virology 65(2): 324-330

Sprokholt J, Helgers LC, Geijtenbeek TB. 2017. Innate immune receptors drive dengue virus immune activation and disease. Future Virology 13(4): 287-305

Sprokholt JK, Kaptein TM, van Hamme JL, Overmars RJ, Gringhuis SI, Geijtenbeek TBH. 2017a. RIG-I-like Receptor Triggering by Dengue Virus Drives Dendritic Cell Immune Activation and TH1 Differentiation. Journal of Immunology 198(12): 4764-4771

Sprokholt JK, Kaptein TM, van Hamme JL, Overmars RJ, Gringhuis SI, Geijtenbeek TBH. 2017b. RIG-I-like receptor activation by dengue virus drives follicular T helper cell formation and antibody production. PLoS Pathogens 13(11): e1006738; 10.1371/journal.ppat.1006738

Strbo N, Vaccari M, Pahwa S, Kolber MA, Fisher E, Gonzalez L, Doster MN, Hryniewicz A, Felber BK, Pavlakis GN, Franchini G, Podack ER. 2011. Gp96 SIV Ig immunization induces 
840 potent polyepitope specific, multifunctional memory responses in rectal and vaginal mucosa.

841 Vaccine. 29(14): 2619-2625

842

843

844

845

846

847

848

849

850

851

852

853

854

855

856

857

858

859

860

861

862

863

864

865

866

867

868

869

870

871

872

873
Strbo N, Romero L, Podack ER, Villasante EF, Edgel KE. 2016. Secreted heat shock protein gp96-Ig vaccine for malaria prophylaxis. Journal of Immunology 196 (1 Supplement): 146.10

Strbo N, Padula L, Fisher E, Wijayalath W, Patterson NB, Huang J, Ganeshan H, O’Neill K, Garcia D, Etobayeva IV, Edgel KA, Podack ER, Sedegah M, Villasante EF. 2020. Secreted heat shock protein gp96-Ig vaccine induces malaria specific intrahepatic CD8 T cell responses. Journal of Immunology 204(1 Supplement): 168.2

Tardif SD, Coleman K, Hobbs TR, Lutz C. 2013. IACUC review of nonhuman primate research. ILAR Journal 54(2): 234-245

Traggiai E, Puzone R, Lanzavecchia A. 2003. Antigen dependent and independent mechanisms that sustain serum antibody levels. Vaccine. 21 (Suppl 2): S35-S37

Viray J, Rolfs B, Smith DG. 2001. Comparison of the frequencies of major histocompatibility (MHC) class-II DQA1 and DQB1 alleles in Indian and Chinese rhesus macaques (Macaca mulatta). Comparative Medicine 51(6): 555-561

Yao Y, Jeyanathan M, Haddadi S, Barra NG, Vaseghi-Shanjani M, Damjanovic D, Lai R, Afkhami S, Chen Y, Dvorkin-Gheva A, Robbins CS, Schertzer JD, Xing Z. 2018. Induction of Autonomous Memory Alveolar Macrophages Requires T Cell Help and Is Critical to Trained Immunity. Cell 175(6): 1634-1650

Ziegler-Heitbrock L. 2014. Monocyte subsets in man and other species. Cellular Immunology 289: $135-139$

Zhang Q, Cao X. 2019. Epigenetic regulation of the innate immune response to infection. Nature Reviews. Immunology 19(7): 417-432

Zmora N, Bashiardes S, Levy M, Elinav E. 2017. The Role of the Immune System in Metabolic Health and Disease. Cell Metabolism 25(3): 506-521 
Figure 1 (on next page)

Monocyte dynamics in peripheral blood.

(A) CD45+CD14+ monocyte frequencies in naïve animals $(n=9)$ and rhesus macaques received DPIV/TDENV-LAV vaccination/DENV-2 challenge $(n=10)(B)$ Frequency and $(C)$ cell counts of HLA-DR+CD14+ monocytes in naïve animals $(n=9)$ and the D/Ad-PfCA vaccinated animals $(n=5)$. Scatter dot plots show values of individual NHPs with mean \pm standard deviation. Asterisks $(*)$ denote significant differences between naïve and the experimental groups at 0.05 alpha level. 


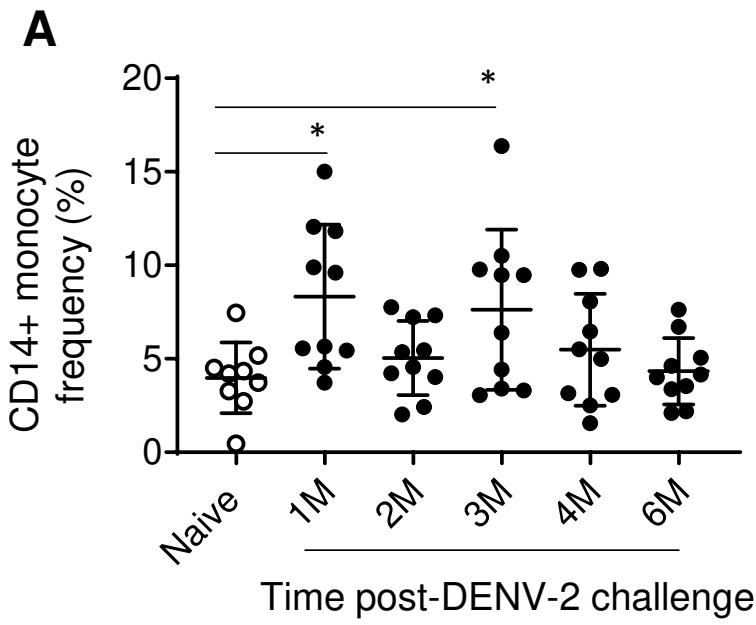

B

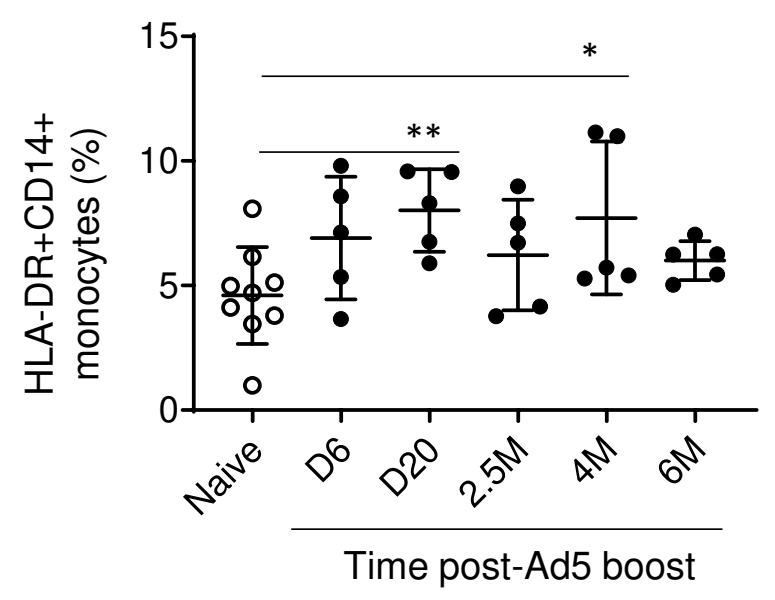

C

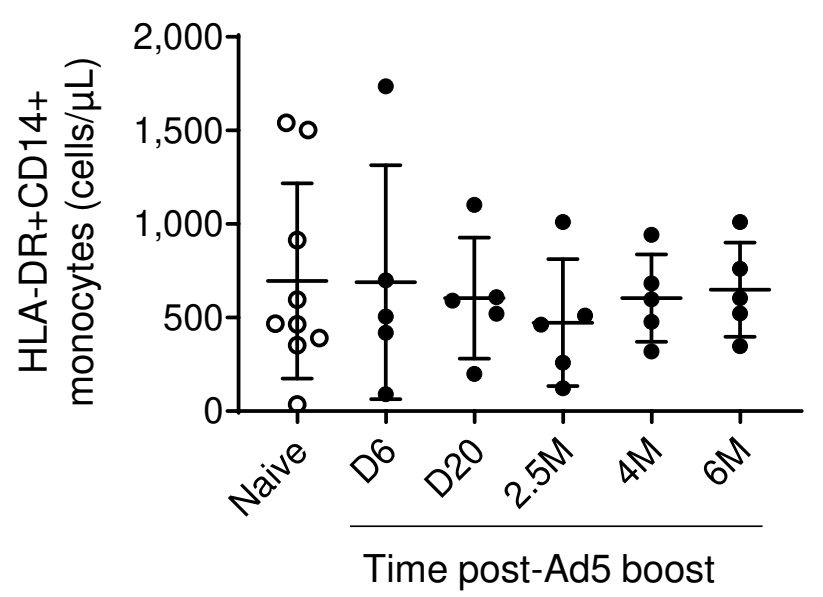


Figure 2

HLA-DR+NKT cells dynamics in peripheral blood.

(A) Frequency and (B) counts of HLA-DR+NKT cells in naïve animals $(n=9)$ and gp96-Ig-PfCA vaccinated animals $(n=5)$. (C) frequency and (D) counts of HLA-DR+NKT cells in naïve animals $(n=9)$ and D/Ad-PfCA vaccinated animals $(n=5)$. Scatter dot plots show values of individual NHPs with mean \pm standard deviation. Asterisks $(*)$ denote significant differences between naïve and the experimental groups at 0.05 alpha level.

A

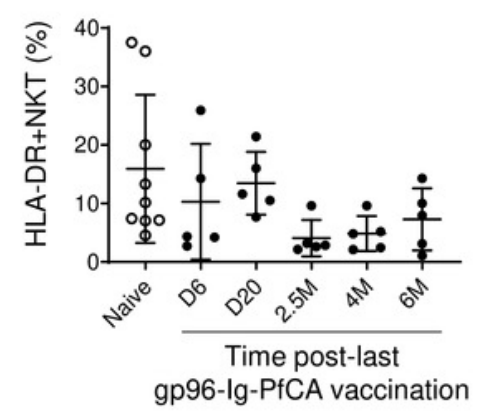

C

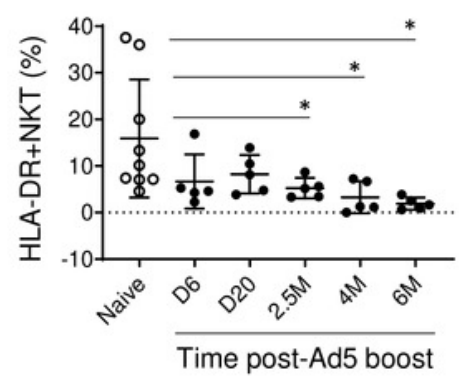

B

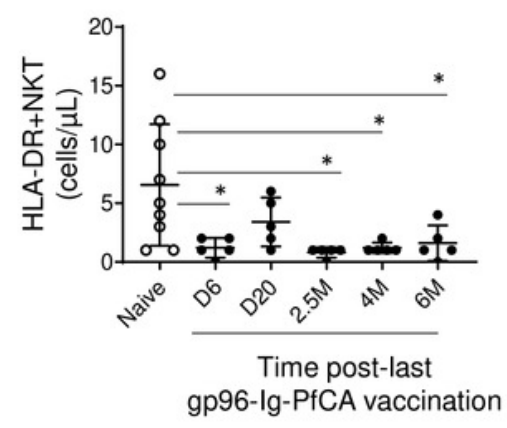

D

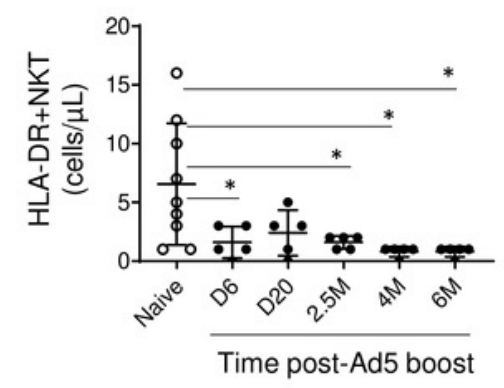




\section{Figure 3}

Circulating concentrations of MCP-1, MIP1- $\alpha$ and IL-12/IL-23p40 cytokines.

(A) naive animals $(n=9)$ and rhesus macaques vaccinated with DPIV/TDENV-LAV followed by DENV-2 challenge $(n=10)$ (B) naïve animals $(n=9)$ and gp96-Ig-PfCA vaccinated animals $(n=5)(C)$ naïve animals $(n=9)$ and D/Ad-PfCA vaccinated animals $(n=5)$. One outlier from the naïve group was removed during IL-12/LL-23p40 analysis $(n=8)$. Scatter dot plots show values of individual NHPs with mean \pm standard deviation. Asterisks $(*)$ denote significant differences between naïve and the experimental groups at 0.05 alpha level.

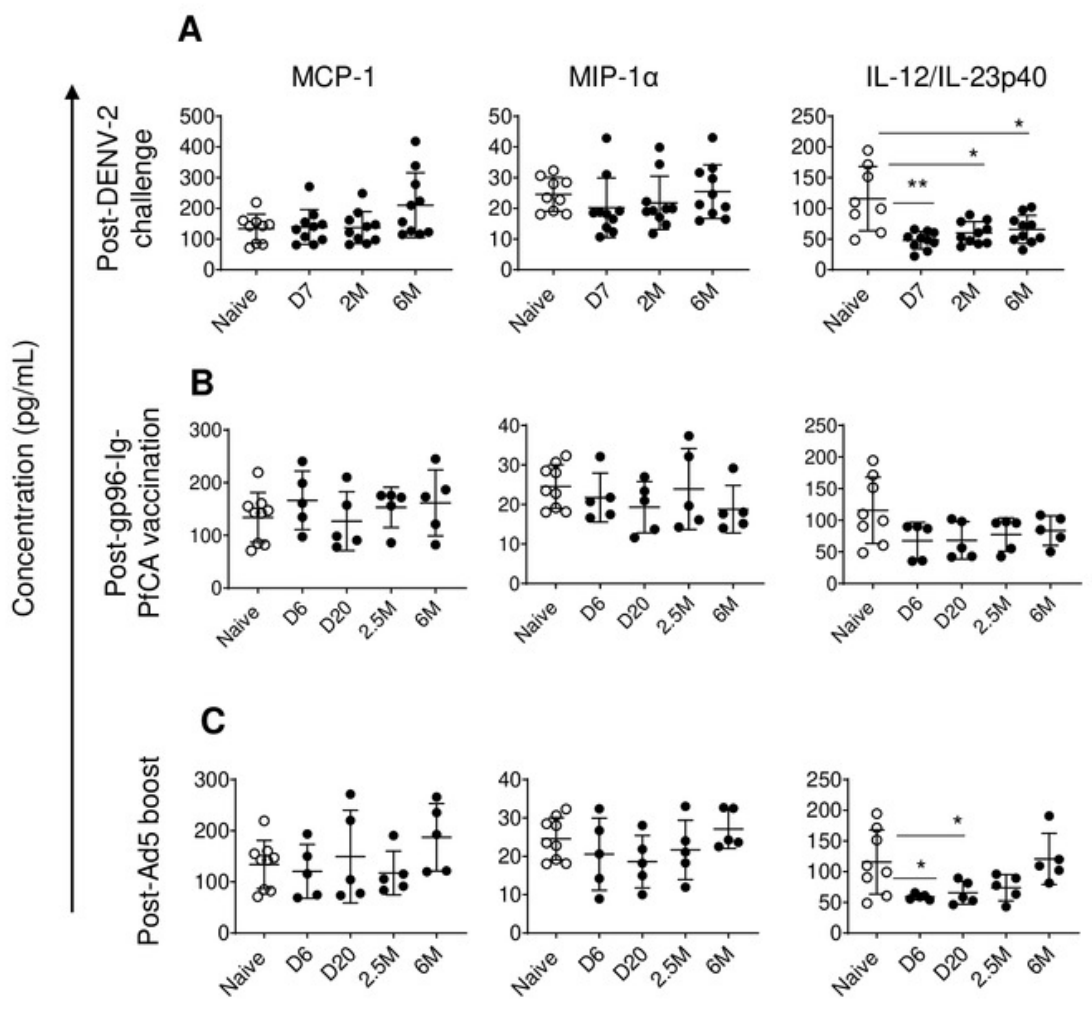


Figure 4

B cell dynamics in peripheral blood.

(A) Frequency and (B) counts of $B$ cells in naïve animals $(n=9)$ and in rhesus macaques vaccinated with DPIV/TDENV-LAV followed by DENV-2 challenge $(n=10)(C)$ frequency and (D) counts of $B$ cells in naïve animals $(n=9)$, gp96-Ig-PfCA vaccinated animals $(n=5)$ and D/AdPfCA vaccinated animals $(n=5)$. Data represent mean \pm standard deviation. Asterisks $(*)$ denote significant differences between naïve and the experimental groups at each time point (0.05 alpha level).

A

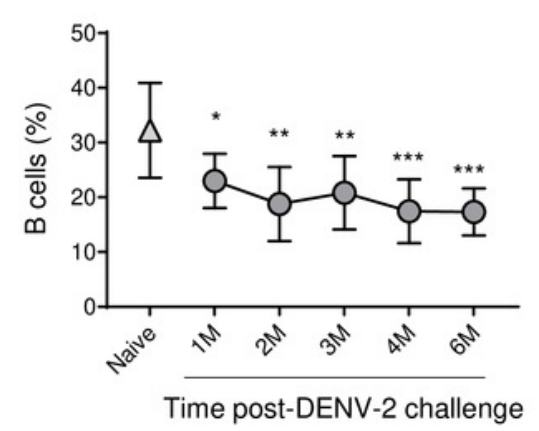

C

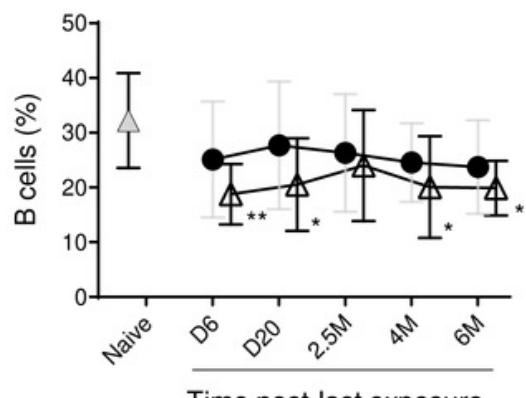

B

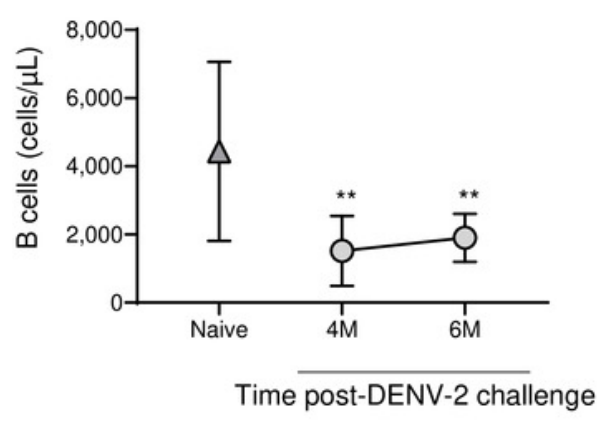

D

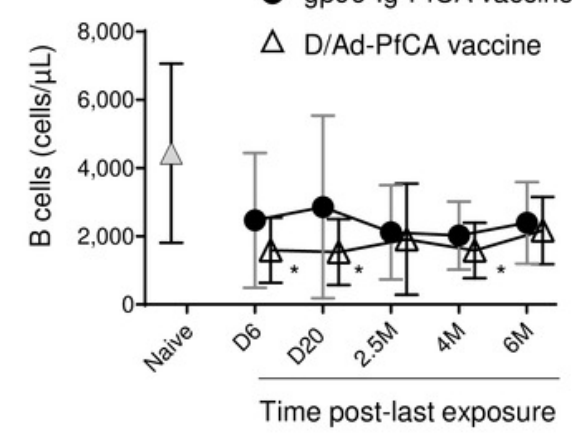


Figure 5

T cell dynamics in peripheral blood.

(A) Cell frequency of $C D 3+$ gated $C D 8+T$ cells $(C D 3+C D 8+)$ and double negative $T$ cells (CD3+CD8-CD4-) in naïve group ( $\mathrm{n}=9)$ and in DPIV/TDENV-LAV vaccinated animals at 1 month post-DENV-2 challenge (B) frequency and (C) counts of CD3+ gated CD4+ T cells $(C D 3+C D 4+)$ in naïve group $(n=9)$ and D/Ad-PfCA vaccinated animals $(n=5)$. Scatter dot plots show values of individual NHPs with mean \pm standard deviation. Asterisks $(*)$ denote significant differences between naïve and the experimental groups at 0.05 alpha level.

A

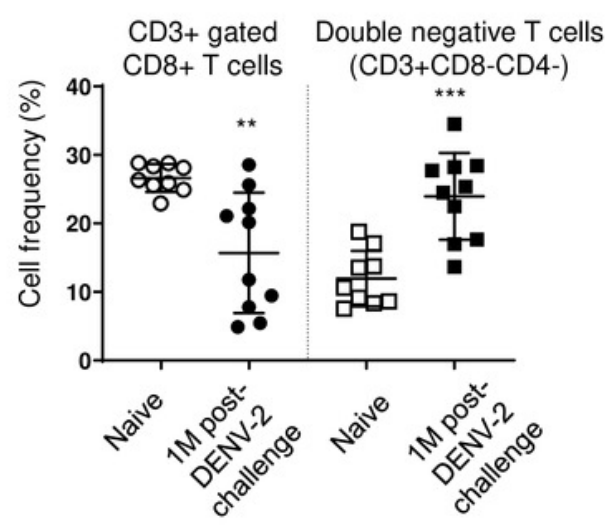

B

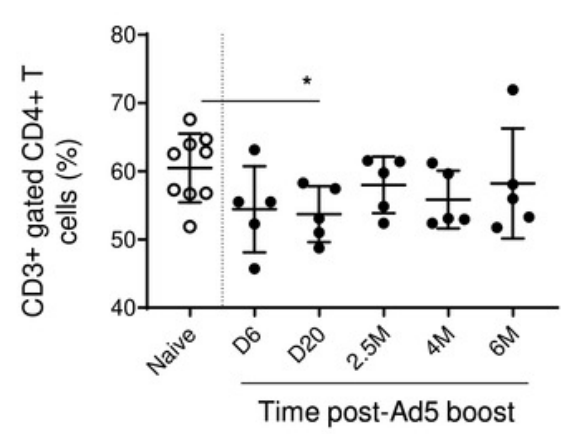

C

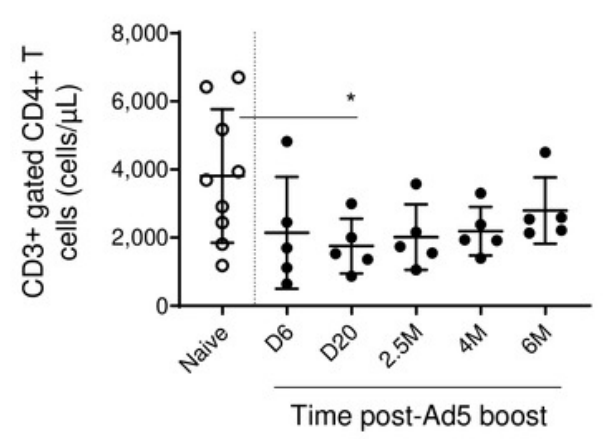




\section{Figure 6}

Dynamics of HLA-DR expressing, activated T cells in peripheral blood.

(A) Frequency and (B) cell counts of activated T cell subsets expressing HLA-DR in naïve $(n=9)$ and gp96-Ig-PfCA vaccinated $(n=5)$ animals. (C) Frequency of activated CD3+T cells expressing HLA-DR (CD45+CD3+HLA-DR+) in naïve animals $(n=9)$ and D/Ad-PfCA vaccinated animals $(n=10)(D)$ Frequency of $C D 3+$ gated, $C D 4+T$ cells expressing HLA-DR $(C D 3+C D 4+H L A-D R+)$ in naïve animals $(n=9)$ and D/Ad-PfCA vaccinated animals. Bar graphs show mean and the standard deviation. Scatter dot plots show values of individual NHPs with mean + standard deviation. Asterisks $(*)$ denote significant differences between naïve and the experimental groups at 0.05 alpha level. 


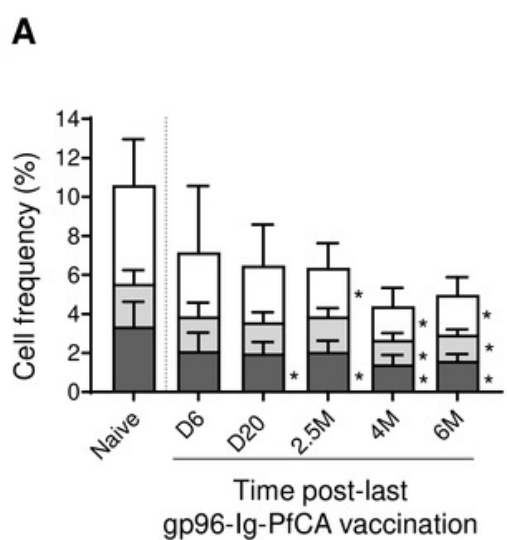

C

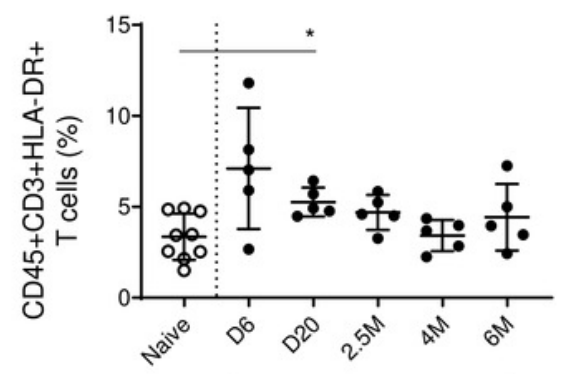

Time post-Ad5 boost

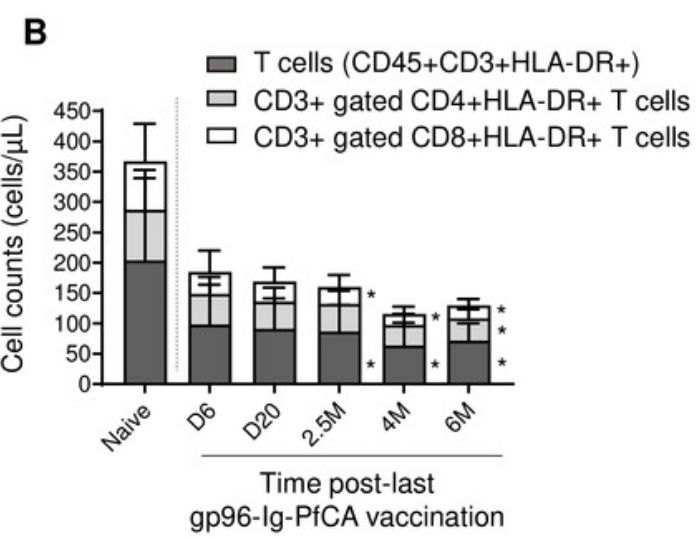

D

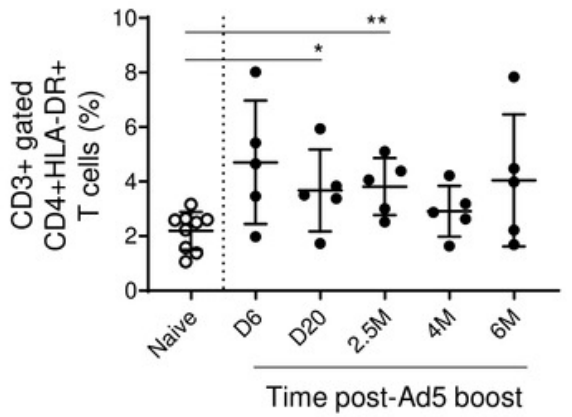




\section{Figure 7}

Correlation analysis and hierarchical clustering of immune cell subsets of naïve and preexposed animals.

Correlograms show the correlation between cell counts (cell/ $\mu \mathrm{L}$ ) of circulating immune cell subsets, within each of the following groups; (A) Naïve $(n=9)$ (B) gp96-Ig-PfCA vaccinated $(n=25)$ and $(C)$ D/Ad-PfCA vaccinated $(n=25)$ animals. Data from naive animals represent only a single time point. For the two vaccinated groups, samples were pooled from the 5 time points post-last exposure ( $n=5 \times 5) ; 6$ days, 20 days, 2.5 months, 4 months and 6 months to capture the immune cell dynamics over 6 months. DENV group was not included in the correlation analysis, due to unavailability of cell count data for all the time points. Positive correlations are displayed in blue and negative correlations in red color. Color intensity and the size of the circle are proportional to the correlation coefficients ( $r$ ). Dendograms display hierarchal relationship between immune cell subsets produced by correlation analysis. Agglomerative hierarchical clustering integrated with complete linkage method (distance Euclidean, Pvclust) was used to build the immune cell clusters. Each leaf of the dendrogram corresponds to one immune cell subset. Closely associated cell subsets are fused into branches. Similarity of the association between two immune cell subsets decreases as height of the fusion increases along the vertical axis. Values at branches are AU p-values (approximately unbiased (AU) probability values (\%) by multiscale bootstrap resampling). Clusters with $\mathrm{AU}>=95 \%$ are considered to be strongly supported by data and highlighted by the red rectangles. We used cell counts calculated based on the cell frequencies of helper $T$ $(C D 45+C D 4+)$ and cytotoxic $T$ cells (CD45+CD8+) directly gated on parent CD45+ leukocyte population, instead of sub-gating on CD3 $+\mathrm{T}$ cell population for correlation analysis and hierarchical clustering (Fig. S1B). 

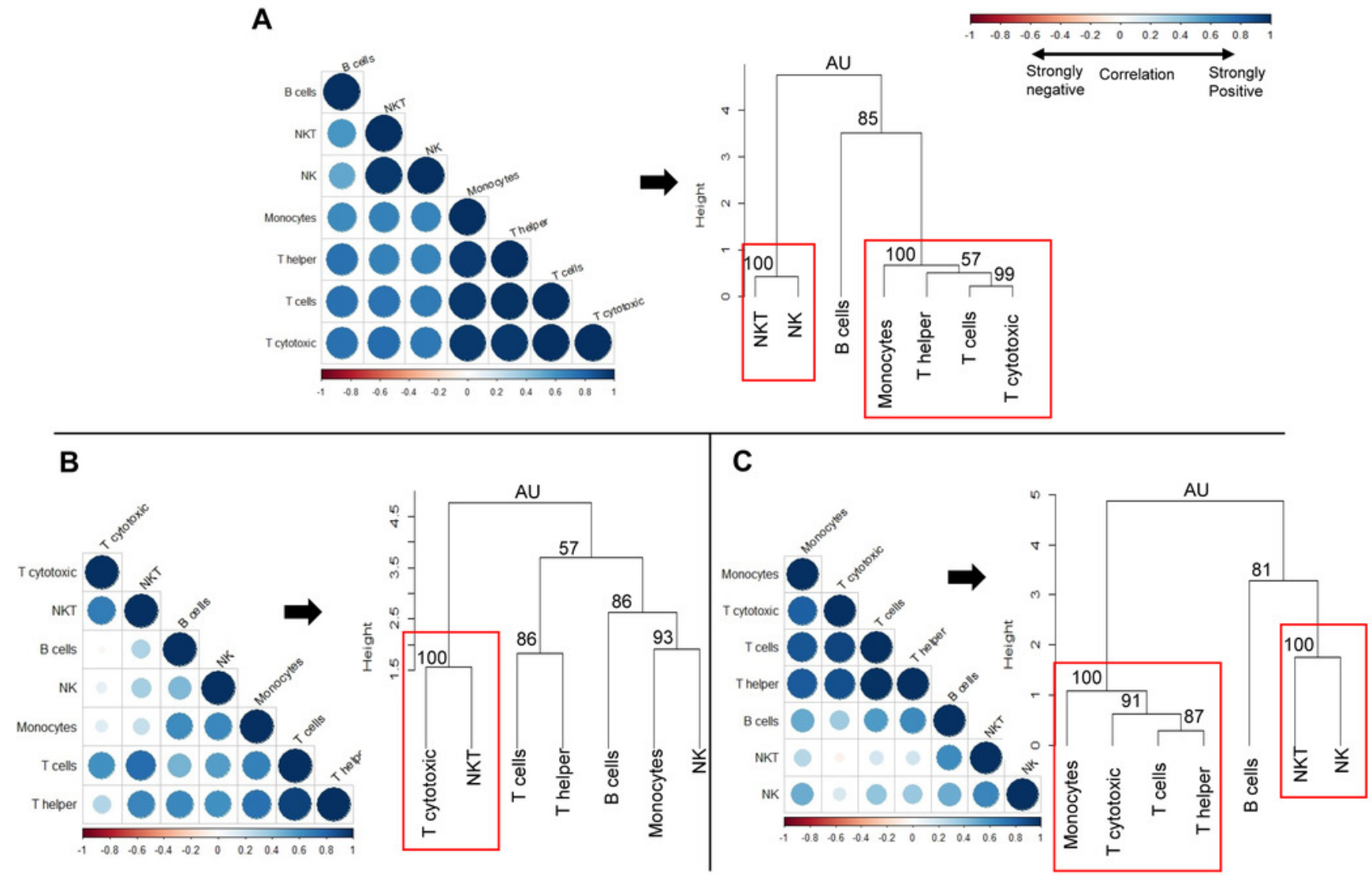